\title{
HONGOS HISTERIOIDES (DOTHIDEOMYCETES, ASCOMYCOTA) DEL BOSQUE TROPICAL CADUCIFOLIO EN EL PARQUE NACIONAL LAGUNAS DE CHACAHUA, OAXACA, MÉXICO
}

\author{
Víctor I. Álvarez ${ }^{1}$, Tania Raymundo y Ricardo Valenzuela ${ }^{1,2}$ \\ ${ }^{1}$ Instituto Politécnico Nacional, Escuela Nacional de Ciencias Biológicas, \\ Departamento de Botánica, Laboratorio de Micología, \\ Prolongación de Carpio y Plan de Ayala, Santo Tomás, \\ Delegación Miguel Hidalgo, 11340 México, D.F., México. \\ ${ }^{2}$ Autor para la correspondencia: rvalenzg@ipn.mx
}

\section{RESUMEN}

Se describen e ilustran cuatro especies de hongos histerioides pertenecientes a la clase Dothideomycetes del phylum Ascomycota, que se encontraron en el bosque tropical caducifolio del Parque Nacional Lagunas de Chacahua, estado de Oaxaca. De éstas, Anteaglonium abbreviatum y Rhytidhysteron rufulum son nuevos registros para la entidad, Psiloglonium simulans se cita por primera vez para México y Gloniella tropicalis se describe como especie nueva para la ciencia.

Palabras clave: especie nueva, Hysteriales, nuevos registros, Pleosporales, taxonomía.

\begin{abstract}
Four species of hysterioid fungi belonging to the class Dothideomycetes of the phylum Ascomycota occurring in the tropical dry forest in the National Park of "Lagunas de Chacahua" in the state of Oaxaca are described and illustrated. Of these, Anteaglonium abbreviatum and Rhytidhysteron rufulum are new records for the state, Psiloglonium simulans is cited for the first time for Mexico and Gloniella tropicalis is described as a species new to science.
\end{abstract}

Key words: Hysteriales, new records, new species, Pleosporales, taxonomy. 


\section{INTRODUCCIÓN}

El Parque Nacional Lagunas de Chacahua tiene una superficie de 14,896 hectáreas y comprende parte del municipio Villa de Tututepec de Melchor Ocampo, distrito de Juquila y se ubica entre las coordenadas extremas $15^{\circ} 57^{\prime} 02.37^{\prime \prime}-16^{\circ} 03^{\prime} 05.96^{\prime \prime}$ latitud norte y $97^{\circ} 31^{\prime} 57.15^{\prime \prime}-97^{\circ} 48^{\prime} 01.01^{\prime \prime}$ longitud oeste (Vargas, 1984; CONANP, 2014). La altitud varía desde el nivel del mar hasta los 200 metros, y presenta épocas de lluvias y secas bien marcadas, el clima predominante es de tipo tropical con lluvias en verano "Aw1" (Vargas, 1984). El bosque tropical caducifolio (o selva baja caducifolia) tiene una extensión de 1269.52 hectáreas, correspondiente a $8.52 \%$ de la superficie del Parque Nacional y regularmente es la vegetación que crece sobre las áreas más cálidas, subhúmedas o semisecas (CONANP, 2014). Las especies presentes son árboles como Amphipterygium adstringens (Schltdl.) Schiede ex Standl. (cuachalalate), Bursera simaruba (L.) Sarg. (palo mulato), Ceiba aesculifolia (Kunth) Britten \& Baker (pochota), Cordia elaeagnoides DC (cobote), Enterolobium cyclocarpum (Jacq.) Grises (parota), Guaiacum coulteri Gray (guayacán), Guazuma ulmifolia Lam. (corazón bonito), Lysiloma microphylla Benth. (minasco), Pithecellobium dulce (Roxb.) Benth. (guamúchil), Prosopis juliflora (Sw.) DC (mezquite) y Tabebuia chrysantha (Jacq.) G. Nicholson (macuil mareño), entre otras (Vargas, 1979; CONANP, 2014).

Los hongos histerioides se caracterizan por presentar ascomas de tipo histerotecio, errumpentes o superficiales en la madera sobre la cual crecen, son de forma alargada o navicular, simples o en ocasiones ramificados, al principio están cerrados, pero en la madurez se abren mediante una fisura longitudinal o varias radiales para formar una línea de dehiscencia, exponiendo el himenio para liberar las esporas, tienen un peridio grueso, carbonáceo; hamatecio formado por seudoparáfisis celulares o trabeculares y ascas bitunicadas con una cámara ocular apical (Sierra-López, 2006; Ulloa y Hanlin, 2006; Kirk et al., 2008). Este grupo morfológico de hongos pertenece a los órdenes Hysteriales, Mytilinidiales, Pattelariales y Pleosporales de la clase Dothideomycetes del Phylum Ascomycota (Kirk et al., 2008; Boehm et al., 2009a,b). En México, los hongos histerioides han sido poco estudiados, y a la fecha solo se conocen ocho especies: 1) Anteaglonium abbreviatum (Schwein.) Mugambi \& Huhndorf de Sonora [Méndez-Mayboca et al., 2010, como Glonium abbreviatum (Schwein.) L.M. Lohman] y Veracruz (Chacón et al., 2014), 2) Gloniopsis praelonga (Schwein.) Underw. \& Earle de Sonora (MéndezMayboca et al., 2008) y Veracruz (Chacon et al., 2014), 3) Hysterium angustatum Alb. \& Schwein de Guerrero (Salinas-Salgado et al., 2012), 4) Hysterium truncatulum 
Cooke \& Peck de Sonora (Méndez-Mayboca et al., 2008), 5) Hysterobrevium mori (Schwein.) E. Boehm \& C.L. Schoch de Oaxaca, Puebla, Sonora, Tamaulipas y Veracruz (Chacón et al., 2014) y de Sonora [Méndez-Mayboca et al., 2010, como Hysterographium mori (Schwein.) Rehm], 6) Oedohysterium insidens (Schwein.) E. Boehm \& C.L. Schoch de Sonora (Méndez-Mayboca et al., 2008, como Hysterium insidens Schwein.) y de Puebla y Sonora (Chacón et al., 2014), 7) Psiloglonium clavisporum (Seaver) E. Boehm, C.L. Schoch \& Spatafora de Veracruz (Chacón et al., 2014) y 8) Rhytidhysteron rufulum (Spreng.) Speg. de Sonora (Méndez-Mayboca et al., 2010), así como de Chiapas, Guerrero, Nuevo León, Puebla, Querétaro, Quintana Roo, Sonora, Tabasco, Tamaulipas y Veracruz (Chacón et al., 2014). El presente trabajo es el primero de una serie que se hará enfocado exclusivamente a los hongos histerioides y tiene como objetivo ampliar la distribución de este grupo de hongos para la micobiota de Oaxaca y de México.

\section{MATERIALES Y MÉTODOS}

En 2011 se realizaron dos exploraciones al Parque Nacional Lagunas de Chacahua y se recolectaron algunos especímenes de hongos histerioides los cuales se caracterizaron en fresco, describiendo los caracteres morfológicos del histerotecio como forma, color, tamaño, consistencia, hábito de crecimiento y hábitat, tamaño de ascas y ascosporas, forma, color y número de células de las ascosporas, y distribución y número de esporas en el asca. Los especímenes se deshidrataron, desinfectaron, etiquetaron y fueron depositados en la colección de hongos del Herbario de la Escuela Nacional de Ciencias Biológicas del Instituto Politécnico Nacional (ENCB). Los ejemplares se determinaron utilizando las claves de Sierra-López (2006) y Boehm et al. (2009a). La clasificación y los nombres están de acuerdo con el trabajo de Boehm et al. (2009a, b). Los términos micológicos utilizados son de acuerdo con Ulloa y Hanlin (2006).

\section{RESULTADOS}

Según Kirk et al. (2008) las cuatro especies estudiadas en este trabajo pertenecen a los géneros Anteaglonium de la familia Anteagloniaceae del orden Pleosporales, Gloniella de la familia Hysteriaceae del orden Hysteriales, Psiloglonium de la familia Gloniaceae del orden Mytilinidiales y Rhytidhysteron de la fami- 
lia Patellariaceae del orden Patellariales, todos de la clase Dothideomycetes del phylum Ascomycota. Sin embargo, Boehm et al. (2009a, b), al realizar un estudio filogenético de los hongos histerioides, encontraron que Gloniella, Psiloglonium y Rhytidhysteron pertenecen a la familia Hysteriaceae del orden Hysteriales. En el presente estudio se seguirá el criterio de Boehm et al. (2009b) por considerarse más actualizado. A continuación se describen las especies encontradas en el bosque tropical caducifolio del Parque Nacional Lagunas de Chacahua del estado de Oaxaca.

Anteaglonium abbreviatum (Schwein.) Mugambi \& Huhndorf, Systematics and Biodiversity 7(4): 460. 2009. Tipo: ESTADOS UNIDOS DE AMÉRICA. Pennsylvania, Northampton County, Bethlehem, L.D. Schweinitz 2094, In: Synopsis Fungorum in America Boreali media degentium. Secundum observationes: 245. 1834. Transactions American Philosophycal Society, New Series 4(2). (Especímen tipo no designado ni se menciona en que herbario se depositó). Fig. 1: A-E.

Histerotecios 0.6-1.0 $\mathrm{mm}$ de largo, 0.4-0.6 $\mathrm{mm}$ de ancho, ovoide a elipsoide, simples, no ramificados, consistencia carbonácea, rectos de extremos obtusos, errumpentes, superficiales, de hábito gregario. Peridio muy grueso, 160-190 $\mu \mathrm{m}$ de grosor, más ancho en la parte media, la base y el extremo apical más delgados, carbonosos, glabros, lisos a ligeramente rugosos, formado por seudoparénquima de células pigmentadas, de forma irregular a subglobosas, 8.8-11.2 $\mu \mathrm{m}$ de diámetro, con paredes gruesas de hasta $1.6 \mu \mathrm{m}$. Seudoparáfisis filamentosas muy delgadas, 0.5 $0.8 \mu \mathrm{m}$ de grosor, hialinas, simples a ramificadas, ligeramente anastomosadas en el ápice formando un epitecio hialino, evanescente. Ascas bitunicadas, cilíndricas, hialinas, 43-65 × 5-8 $\mu \mathrm{m}$, octospóricas. Ascosporas 6.4-7.2 × 2.4-3.2 $\mu \mathrm{m}$, hialinas, bicelulares, de ápices redondeados, paredes delgadas, lisas, célula superior más ancha que la inferior, fuertemente constreñidas en el septo, irregularmente biseriadas en el asca.

Material estudiado. MÉXICO. Oaxaca, Distrito de Juquila, Municipio de San Pedro Tututepec, Parque Nacional Lagunas de Chacahua, km 4 del camino de terracería Los Azufres - La Grúa, bosque tropical caducifolio a nivel del mar, noviembre 12, 2011, R. Valenzuela 14596 (ENCB); T. Raymundo 4096 (ENCB).

Hábitat. Gregario, sobre madera en descomposición de angiospermas en el bosque tropical caducifolio y ocasiona una pudrición blanda. 

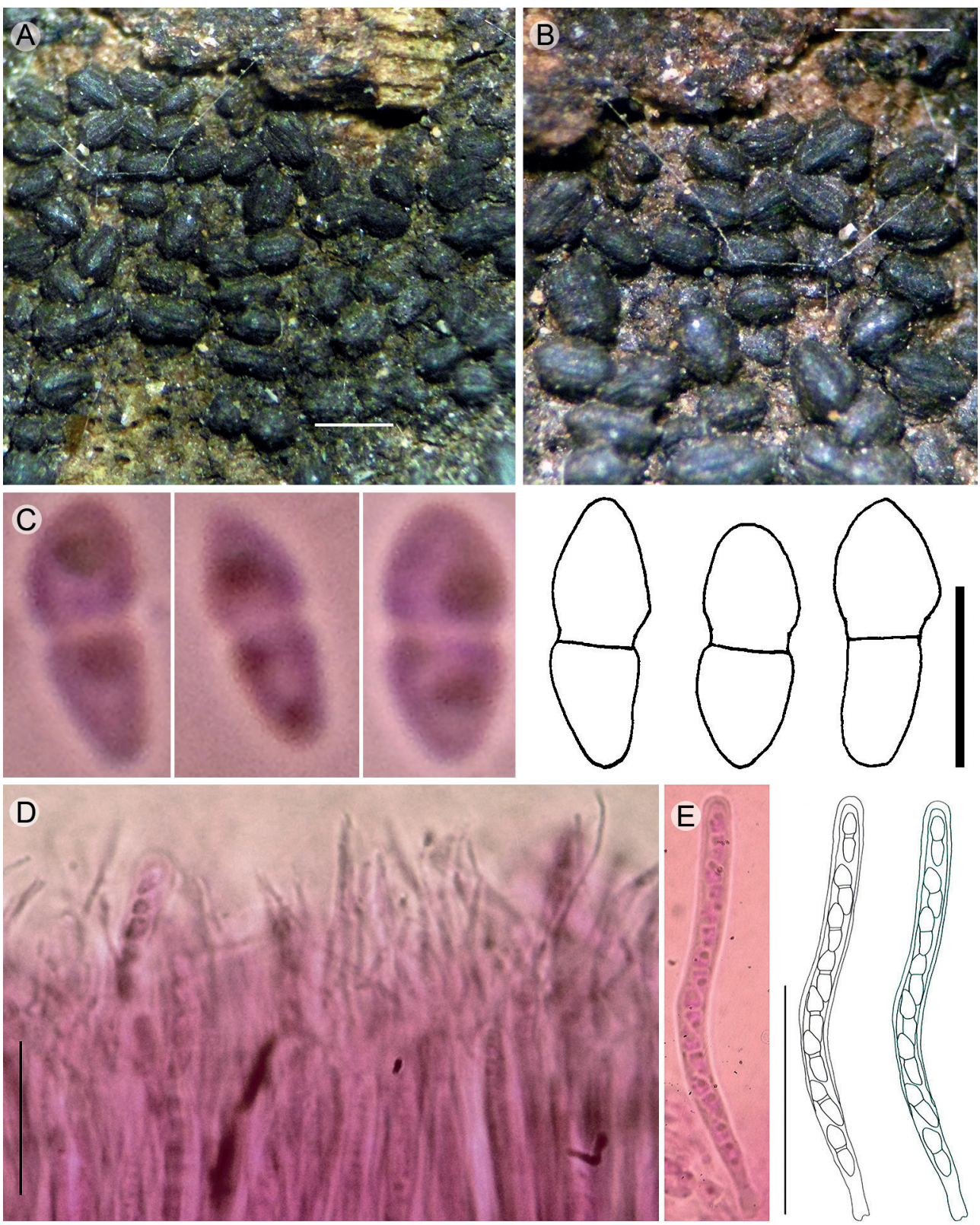

Fig.1. Anteaglonium abbreviatum. A-B. histerotecios; C. ascosporas; D. ascas en el corte transversal del histerotecio; E. Ascas y ascosporas. Escala de la barra $A-B=1 \mathrm{~mm}, \mathrm{C}=4 \mu \mathrm{m}$, $\mathrm{D}=20 \mu \mathrm{m}, \mathrm{E}=30 \mu \mathrm{m}$. 
Esta especie se caracteriza por los histerotecios con ápices truncados, gregarios y por la presencia de un subículo o costra negra que obscurece el sustrato. Especies afines son A. globosum Mugambi y Huhndorf y A. parvulum (W.R. Gerard) Mugambi y Huhndorf, por tener ascosporas similares en forma y tamaño. La primera se separa por formar histerotecios con ápices redondeados dispuestos sobre un subículo o costra que forma un tomento en la base de los ascomas, y porque producen un pigmento soluble de color verde con $\mathrm{KOH}$. La segunda especie se separa por los histerotecios con ápices agudos y por la carencia de subículo. Esta especie presenta amplia distribución y ha sido citada de varios países de América, Europa, Asia, África y Oceanía por Boehm et al. (2009a) y Almeida et al. (2014). Fue citada de México por Méndez-Mayboca et al. (2010) de Sonora y por Chacón et al. (2014) de Veracruz y Sonora, quienes la mencionaron de un bosque tropical caducifolio. En el presente trabajo se cita por primera vez para el estado de Oaxaca del mismo tipo de vegetación.

Gloniella tropicalis V. I. Álvarez, Raymundo \& R. Valenz. sp. nov. Tipo: MÉXICO. Oaxaca, Parque Nacional Lagunas de Chacahua, $\mathrm{km} 4$ del camino de terracería Los Azufres - La Grúa, 12 noviembre 2011, T. Raymundo 4093 (Holotipo: ENCB). Fig. 2: A-F. MB 816523

Hysterothecia aggregata, erumpentia, navicularia, recta vel flexuosa, carbonacea, 0.5-1 mm longa, 0.2-0.3 mm lata, sulco conspicuos, levis apertus, epithecius expositus. Peridium 28-56 $\mu \mathrm{m}$ crassum. Pseudoparaphyses non ramosae vel ramosae, 1-1.5 $\mu \mathrm{m}$ latae, sursum magis crassitunicatae, supra ascos ramosae epithecium formantes, pigmentatum, 16-24 $\mu \mathrm{m}$ crasum. Asci cylindrici vel clavati, stipite levis sinuosis, bitunicati, (81-)96-110 × 9.6-12.8 $\mu \mathrm{m}$, octosporis; ascosporae irregulariter biseriatae, rectae vel levis curvatae, $16-19.2 \times 7.2-8 \mu \mathrm{m}$, tenuitunicatae, hyalinae, 3 -septatae, septis transversis divisae, cingulatis. Ad lignis in angiospermis, in sylvis tropical caducifoliae.

Histerotecios 0.5-1.5 mm de largo y 0.2-0.6 mm de ancho, naviculares, alargados, simples, algunos bifurcados, carbonáceos, rectos a flexuosos, con los extremos agudos, pero algunos llegan a ser obtusos, errumpentes, superficiales, gregarios. Surco longitudinal, conspicuo, ligeramente abierto, exponiendo el epitecio negro. Peridio 28-56 $\mu \mathrm{m}$ de grosor, adelgazándose hacia el extremo apical, base y parte media más gruesas, carbonáceo, glabro y liso, formado por un seudoparénquima, de células pigmentadas, marrón obscuro a negras, con paredes muy gruesas (hasta 
$2.4 \mu \mathrm{m}$ de grosor), globosas, alargadas a irregulares en forma, 6.4-8 $\mu \mathrm{m}$ de diám. Seudoparáfisis filamentosas, septadas, hialinas, simples o ramificadas, 1-1.6 $\mu \mathrm{m}$ de diám., en el ápice hasta $2.4 \mu \mathrm{m}$ de ancho, anastomosadas, formando un epitecio con pigmentos verde oliváceo oscuro, 16-24 $\mu \mathrm{m}$ de grosor. Ascas (81-)96-110 × 9.6-12.8 $\mu \mathrm{m}$, bitunicadas, de paredes gruesas, hialinas, octospóricas, cilíndricas a clavadas, con la base sinuosa. Ascosporas 16-19.2 $\times 7.2-8 \mu \mathrm{m}$, elipsoides a oblongas, con el centro más ancho y extremos redondeados, rectas a ligeramente curvadas, hialinas, gutuladas, con tres septos transversales, cingulados, segunda célula más grande, de paredes delgadas, lisas, irregularmente biseriadas en el asca.

Material estudiado. Paratipo: MÉXICO. Oaxaca, Distrito de Juquila, municipio de San Pedro Tututepec, Parque Nacional Lagunas de Chacahua, km 4 del camino de terracería Los Azufres - La Grúa, 12 noviembre 2011, R. Valenzuela 14593 (ENCB).

Etimología. El epíteto específico tropicalis debe su nombre al tipo de vegetación donde se encontró la especie.

Hábitat. Esta especie es gregaria y se encontró en una cerca, sobre madera descortezada de angiospermas en un bosque tropical caducifolio a nivel del mar y ocasiona una pudrición blanda.

Esta especie se ubica en el género Gloniella por presentar ascosporas hialinas y septos transversales (fragmosporas) y ascomas de tipo histerotecioide. Se separa de los géneros Anteaglonium, Glonium y Psiologlonium por que tienen ascosporas bicelulares (didimosporas); de Hysterium y Oedohysterium que presentan ascosporas pigmentadas con tres septos transversales; de Hysterobrevium, Hysterographium y Gloniopsis por sus ascosporas con septos transversales y longitudinales (dictiosporas) hialinas o pigmentadas y de Rhytidhysteron por sus ascomas apoteciales y ascosporas pigmentadas (Boehm et al., 2009a). Gloniella tropicalis se caracteriza por tener histerotecios muy pequeños, de hasta $1 \mathrm{~mm}$ de largo, con una hendidura longitudinal conspicua y semiabierta en algunos ascomas, las ascosporas son elipsoidales a oblongas, hialinas, con 4 células, la segunda célula es más ancha y grande, lo que les da a algunas esporas la forma ligeramente curvada, mientras que otras se observan rectas. Especies afines son G. corticola A. Pande \& V.G. Rao y G. typhae (Fuckel) Sacc. que presentan ascosporas con 3 septos transversales, pero se separan por tener esporas menores a $15 \mu \mathrm{m}$; la primera con una distribución en 

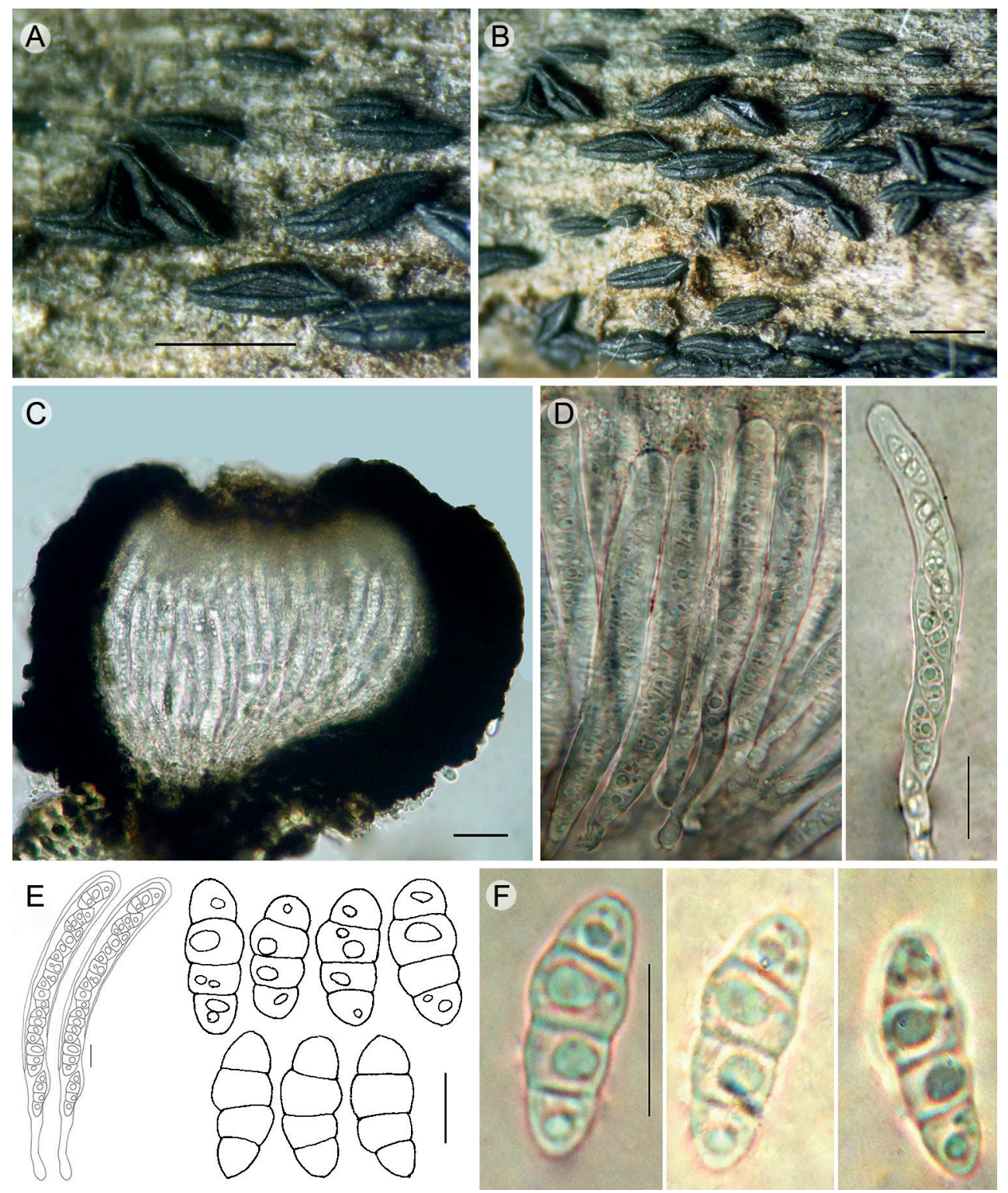

Fig. 2. Gloniella tropicalis. A-B. histerotecios; C. himenio en corte transversal del histerotecio; D-E. ascas; F. ascosporas. Escala de la barra $A-B=1 \mathrm{~mm}, \mathrm{C}=30 \mu \mathrm{m}, \mathrm{D}=20 \mu \mathrm{m}, \mathrm{E}-\mathrm{F}=8 \mu \mathrm{m}$. 
la India y la segunda se ha encontrado en Europa, Argentina y Chile (Boehm et al., 2009a; Messuti y Lorenzo, 2009). Otra especie afín es G. adianti (Kunze) Petr. por el largo de las ascosporas y numero de septos, pero tiene un diámetro menor (3-6 $\mu \mathrm{m})$ que la especie mexicana; además, difiere en la forma subglobosa y ancho de las ascas $(14-17 \mu \mathrm{m})$ y el arreglo irregular de las ascosporas dentro del asca. Aunado a esto crece sobre frondas caídas de helechos en zonas templadas de Alemania, España e Inglaterra (Dennis, 1981). También ocurre en Brasil, sobre corteza no identificada, creciendo en bosque tropical seco (Almeida et al., 2014). Otras especies con ascosporas con tres septos son G. abietina Sid., G. lapponica (P. Karst.) Sacc., G. araucana Speg. y G. chilensis Speg., pero se separan porque las esporas son fusiformes y más grandes ( $>24 \mu \mathrm{m})$; además, presentan hospederos con distribución en las zonas templadas del Hemisferio Norte (las dos primeras) o Sur (las dos últimas). Las demás especies del género se separan por tener esporas de diferente tamaño y con mayor número de septos, además de que solo se conocen de hospederos específicos con distribución templada. Los especímenes mexicanos presentan caracteres morfológicos y ecológicos suficientes para separarla de las demás especies del género Gloniella y se describe como nueva especie, la cual se conoce de la localidad tipo sobre madera descortezada de bosque tropical caducifolio en el estado de Oaxaca. Sin embargo, puede tener una mayor distribución porque los bosques tropicales caducifolios están ampliamente distribuidos en las costas del pacífico mexicano.

Psiloglonium simulans (W.R. Gerard) E. Boehm, C.L. Schoch \& Spatafora, Mycological Research 113(4): 469. 2009. Tipo: ESTADOS UNIDOS DE AMÉRICA. New York, Poughkeepsie. W.R. Gerard s.n. (holotipo: FH). Fig. 3: A-E.

= Glonium simulans W.R. Gerard, Bull. Torrey Bot. Club 6(14): 78. 1876.

Histerotecios 1-2.5 $\mathrm{mm}$ de largo y 0.25-0.4 $\mathrm{mm}$ de ancho, alargados a fusoides, flexuosos, agregados o solitarios, simples, nunca ramificados, con los extremos ligeramente romos, sésiles, superficiales. Surco longitudinal conspicuo, siempre cerrado. Peridio 65-84 $\mu \mathrm{m}$ de grosor, ligeramente más ancho en la parte media, carbonoso, glabro y estriado longitudinalmente, compuesto por un seudoparénquima de células alargadas, de forma irregular, 8-10.4 x $4.8 \mu \mathrm{m}$, pared gruesa de $1.6 \mu \mathrm{m}$, pigmentadas de color marrón oscuro. Seudoparáfisis filamentosas, sin septos, hialinas, ramificadas dicotómicamente, 0.8-1.6 $\mu \mathrm{m}$ de diámetro. Ascas bitunicadas, 95-118($127) \times 9.5-11.4 \mu \mathrm{m}$, hialinas, cilíndricas, octospóricas, de pared gruesa. Ascosporas (13.6-)14-16(-18) × 4.8-5.6 $\mu \mathrm{m}$, bicelulares, hialinas, marrón amarillento pálidas con 
la edad, cinguladas en el septo, ligeramente curvadas, la célula superior más ancha que la inferior, lisas, de pared delgada, célula apical y basal con el extremo ligeramente romo, uniseriadas en el asca.

Material estudiado. MÉXICO. Oaxaca, Distrito de Juquila, Municipio de San Pedro Tututepec, Parque Nacional Lagunas de Chacahua, km 4 del camino de terracería Los Azufres - La Grúa, bosque tropical caducifolio a nivel del mar, 12 noviembre 2011, T. Raymundo 4094 (ENCB).

Hábitat. Gregario, en madera en descomposición no identificada de angiospermas de bosque tropical caducifolio a nivel del mar; produce una pudrición blanda.

Psiloglonium simulans se define por los histerotecios superficiales, flexuosos estrechamente agregados, carentes de un arreglo linear paralelo y por el tamaño y forma de las ascosporas. Una especie afín por el tamaño de las esporas es $P$. lineare (Fr.) Petr., pero se separa por formar histerotecios inmersos con un arreglo linear paralelo, además de que las ascosporas presentan extremo apical muy redondeado (Boehm et al., 2009b), mientras que en P. simulans son ligeramente romos. Otra especie afín es $P$. clavisporum (Seaver) E.W.A. Boehm, C.L. Schoch \& J.W. Spatafora, pero se separa por tener las esporas más grandes [16-18(20) x 5-6 $\mu \mathrm{m}]$ y de forma clavada-fusoides. Esta especie fue referida con una distribución cosmopolita por Boehm et al. (2009a), mencionándola de Chile, Estados Unidos de América y Japón. En el presente trabajo se registra por primera vez para México de un bosque tropical caducifolio.

Rhytidhysteron rufulum (Spreng.) Speg. Anales Sociedad Cientifica Argentina 90(1-6): 177, 1921. Tipo: PUERTO RICO, C. Sprengel s.n., In: Plantarum Cryptogamicarum Tropicarum Pugillus: 50. 1820. Kongl. Vetenskaps Academiens Handlingar (Especímen tipo no designado ni se menciona en que herbario se depositó). Fig. 4: A-H.

Ascoma histerotecial a apotecial, cuando forma histerotecios, éstos 1-4 mm de largo y 0.5-1 mm de ancho, naviculares, alargados, rectos o flexuosos cuando jóvenes, con los extremos agudos, algunos son triradiados, negros, carbonáceos, errumpentes, superficiales, gregarios, raramente solitarios, cuando maduros o con la humedad abren de forma irregular a discoidal, tomando la forma apotecial, 2-4 mm de diám., mostrando el epitecio de color naranja a naranja 

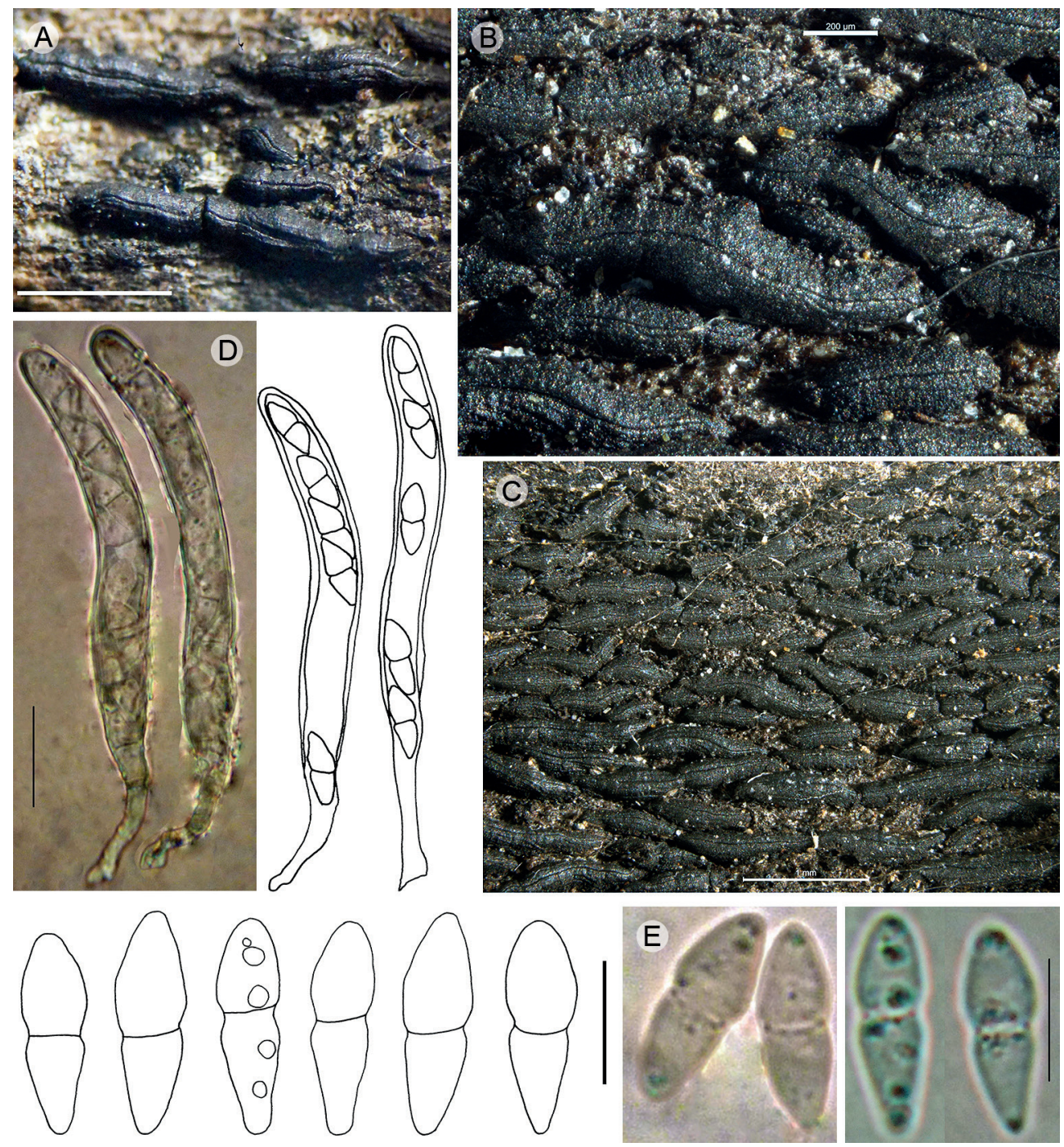

Fig. 3. Psiloglonium simulans. A-C. histerotecios; D. ascas; E. ascosporas. Escala de la barra A y $\mathrm{C}=1 \mathrm{~mm}, \mathrm{~B}=200 \mu \mathrm{m}, \mathrm{D}-\mathrm{E}=8 \mu \mathrm{m}$.

rojizo, con los márgenes estriados, involutos. Surco longitudinal conspicuo, con el margen transversalmente estriado. Peridio 40-72 $\mu \mathrm{m}$ de grosor, adelgazándose hacia el extremo basal y la parte media, parte apical más gruesa, carbonosa, glabra, compuesto por células seudoparenquimáticas globosas a subglobosas, 
9.6-12 × 8-8.8 $\mu \mathrm{m}$, de paredes gruesas, 1.6-2.4 $\mu \mathrm{m}$ de grosor. Seudoparáfisis filamentosas, septadas, hialinas, ramificadas hacia el ápice, 1.6-2.4 $\mu \mathrm{m}$ de diámetro, fuertemente ensanchadas hacia el extremo apical (capitadas) hasta 3.2-4.0 $\mu \mathrm{m}$ de ancho, fuertemente anastomosadas por encima de las ascas formando un epitecio naranja, 25.6-32 $\mu \mathrm{m}$ de grosor, el cual se vuelve violeta al reaccionar con $\mathrm{KOH} \mathrm{5 \%}$. Ascas 142.5-169.7 × 12-14.4 $\mu \mathrm{m}$, bitunicadas, uniseriadas, de paredes gruesas, cilíndricas, hialinas, octospóricas, con la base sinuosa. Ascosporas 22.4-30.4 × 8-9.6 $\mu \mathrm{m}$, elipsoides a oblongas, fusiformes, ligeramente curvadas, de pared gruesa y lisa, pigmentadas de marrón a marrón rojizo, con tres septos transversales, cingulados.

Material estudiado. MÉXICO. Oaxaca, Distrito de Juquila, Municipio de San Pedro Tututepec, Parque Nacional Lagunas de Chacahua, km 5 del camino de terracería Los Azufres - La Grúa, bosque tropical caducifolio a nivel del mar, 12 noviembre 2011, R. Valenzuela 14604 (ENCB); ibid., T. Raymundo 4104 (ENCB); km 7.2 del camino de terracería Los Azufres - La Grúa, bosque tropical caducifolio y matorral xerófilo a nivel del mar, 12 noviembre 2011, R. Valenzuela 14615, 14618, 14619 (ENCB); ibid., T. Raymundo 4115, 4118,4119 (ENCB); ibid., K. Victoria 24; C. Salinas 34 (ENCB).

Hábitat. Esta especie crece gregaria sobre madera de angiospermas no determinada y en descomposición en bosque tropical caducifolio, ocasionando una pudrición blanda.

Rhytidhysteron rufulum se caracteriza por presentar la típica forma histerioide cuando es joven y posteriormente, al madurar llega a ser apotecioide, pero éste en condiciones de baja humedad, el margen colapsa y nuevamente adquiere la forma histerioide o triangular, triradiada (Samuels y Müller, 1979). MéndezMayboca et al. (2010) reportan la coloración violeta en el epitecio al reaccionar con hidróxido de potasio 5\%. Con base en las características anteriormente mencionadas, el material revisado concuerda con las descripciones previas de $R$. rufulum. Una especie similar es $R$. hysterinum (Dufour) Samuels \& E. Müll.; sin embargo, las esporas presentan sólo un septo transverso y ha sido citado únicamente de Europa. Rhytidhysteron opuntiae (J. G. Br.) M. E. Barr se diferencia por presentar esporas con tres septos transversales pero con uno longitudinal formando dictiosporas y ha sido citado de África y Estados Unidos. Por otro lado, se considera que es importante realizar una revisión exhaustiva de Rhytidhysteron rufulum, ya que 

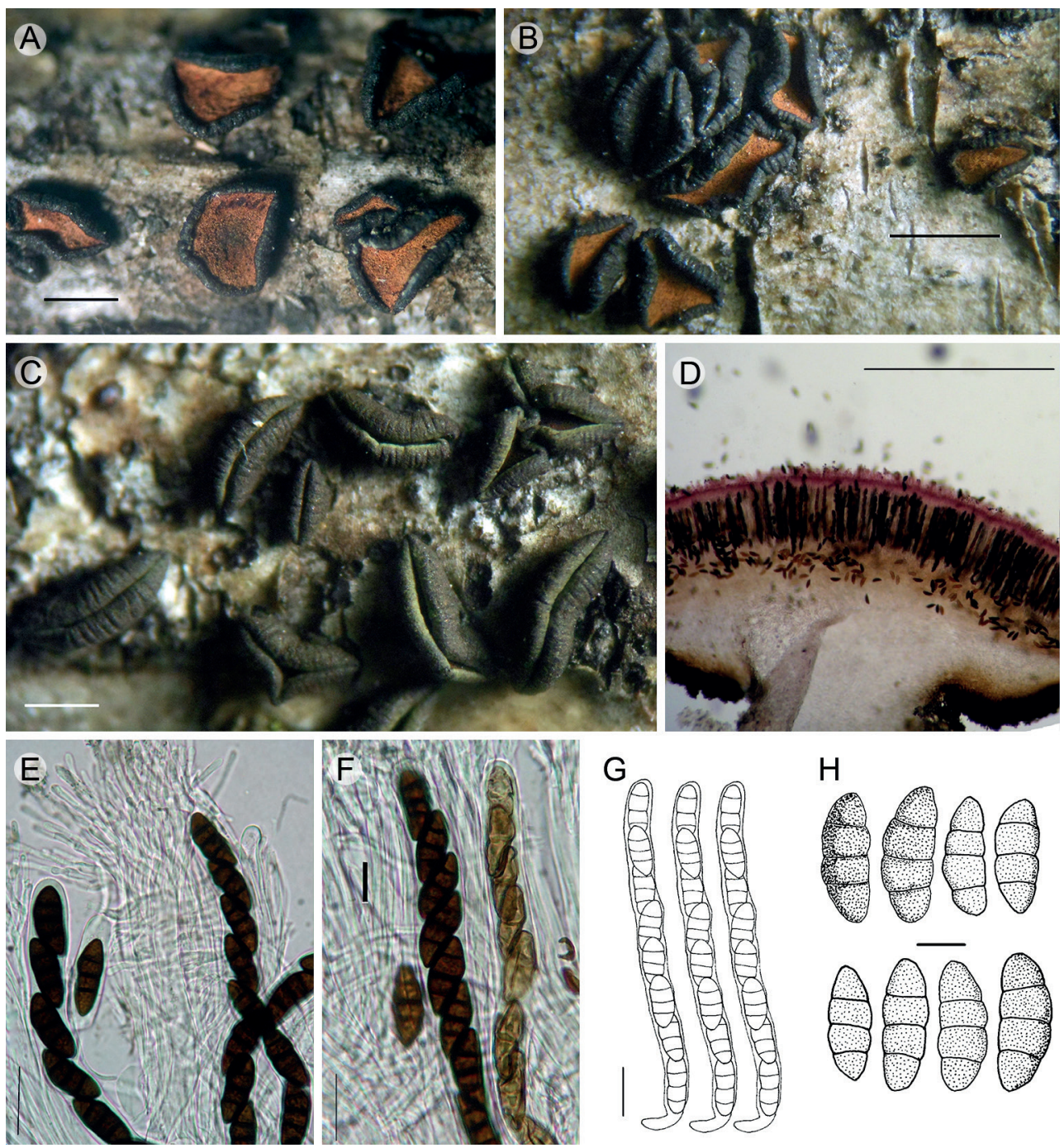

Fig. 4. Rhytidhysteron rufulum. A-B. ascostromas apotecioides; C. ascostromas histerotecioides; D. corte transversal del ascostroma; E-G. ascas y ascosporas; H. ascosporas. Escala de la barra A-C=2 mm, D=280 $\mu \mathrm{m}, \mathrm{E}-\mathrm{F}=15 \mu \mathrm{m}, \mathrm{G}-\mathrm{H}=8 \mu \mathrm{m}$.

de acuerdo con Murillo et al. (2009) puede ser parte de un complejo de especies. Samuels y Müller (1979) la determinan de amplia distribución en zonas tropicales y subtropicales de América, incluido México. Chen y Hsieh (1996) indican que es una especie frecuente en zonas tropicales y lo citan para Taiwan. Sierra-López 
(2006) la menciona de Cataluña. Murillo et al. (2009) la citan de amplia distribución en Costa Rica, e incluyen ejemplares de Estados Unidos, Puerto Rico y Venezuela. Almeida et al. (2014) la mencionan de Argentina, Brasil, China, Costa Rica, Cuba, Dominica, Estados Unidos de América, Filipinas, Francia, Ghana, India, Islas Cook, Jamaica, Japón, Kenia, Malasia, Micronesia, Nueva Guinea, Nueva Zelanda, Puerto Rico, Sierra Leone, Tanzania y Tonga. En México ha sido citado por Méndez-Mayboca et al. (2010) de Sonora y por Chacón et al. (2014) de Chiapas, Guerrero, Nuevo León, Puebla, Querétaro, Quintana Roo, Sonora, Tabasco, Tamaulipas y Veracruz y en el presente trabajo se registra por primera vez para Oaxaca.

\section{DISCUSIÓN}

El presente trabajo refleja la gran diversidad y amplia distribución que tienen las especies de hongos histerioides en México, porque se incrementa a 10 especies conocidas de este grupo de hongos para México. De éstas, ocho proceden del bosque tropical caducifolio resultando en que éste sea un tipo de vegetación con alta riqueza en especies de este grupo de hongos. Además, se describe una especie nueva (Gloniella tropicalis) y un nuevo registro para el país (Psiloglonium simulans). Por otro lado, el estado de Oaxaca pasa a segundo lugar junto con Veracruz en número de especies conocidas de histerioides con cinco, después de Sonora que tiene siete especies. Con menor diversidad están Puebla con tres, Guerrero y Tamaulipas con dos y Nuevo León, Querétaro, Quintana Roo y Tabasco con una especie.

\section{AGRADECIMIENTOS}

Los autores agradecen al IPN el apoyo financiero otorgado mediante el proyecto SIP-20150540, SIP-20151530, SIP-20161164 y SIP-20161166. Tania Raymundo agradece al CONACYT el apoyo otorgado para realizar de 2013 al 2015 dos estancias Posdoctorales en el Posgrado de la Maestría en Biociencias de la ENCB del IPN. Álvarez agradece al IPN el apoyo por la Beca otorgada del Programa Institucional de Formación de Investigadores (antes PIFI, ahora BEIFI). Ricardo Valenzuela reconoce el apoyo otorgado por la COFAA e Instituto Politécnico Nacional a sus investigaciones. 


\section{LITERATURA CITADA}

Almeida, D. A. C., L. F. P. Gusmão y A. N. Miller. 2014. Brazilian Semi-Arid Ascomycetes I: New and interesting records of hysteriaceous ascomycetes. Mycosphere 5(2): 379391.

Boehm, E. W. A., G. K. Mugambi, A. N. Miller, S. M. Huhndorf, S. Marincowitz, J. W. Spatafora y C. L. Schoch. 2009a. A molecular phylogenetic reappraisal of the Hysteriaceae, Mytilinidiaceae and Gloniaceae (Pleosporomycetidae, Dothideomycetes) with key to the world species. Studies in Mycology 64: 49-83.

Boehm, E. W. A., C. L. Schoch y J. W. Spatafora. 2009b. On the evolution of the Hysteriaceae and Mytilinidiaceae (Pleosporomycetidae, Dothideomycetes, Ascomycota) using four nuclear genes. Mycological Research 113: 461-479.

Chacón, S., F. Tapia y M. Esqueda. 2014. New records of Dothideomycetes from Mexico. Mycotaxon 128: 147-157.

Chen, C. Y. and W. H. Hsieh. 1996. Two new species and some new records of ascomycetes from Taiwan. Botanical Bulletin of Academia Sinica 37: 219-227.

CONANP. 2014. Programa de Manejo Parque Nacional Lagunas de Chacahua. Secretaría de Medio Ambiente y Recursos Naturales. Comisión Nacional de Áreas Naturales Protegidas. México, D.F., México. 217 pp.

Dennis, R. W. G. 1981. British Ascomycetes. Ed. J. Cramer. Vaduz, Liechtenstein. 585 pp.

Kirk, P. M., P. F. Cannon, D. W. Minter y J. A. Stalpers. 2008. Ainsworth \& Bisby's Dictionary of the Fungi. 10th ed. CAB International. Wallingford, UK. 771 pp.

Méndez-Mayboca, F., S. Chacón, M. Esqueda y M. L. Coronado. 2008. Ascomycetes of Sonora, México, 1: The Ajos-Bavispe National forest Reserve and Wildlife Refuge. Mycotaxon 103: 87-95.

Méndez-Mayboca, F., J. Checa, M. Esqueda y S. Chacón. 2010. New records of Loculoascomycetes from natural protected areas in Sonora, Mexico. Mycotaxon 111: 19-30.

Messuti, M. I. y L. E. Lorenzo. 2009. Taxonomic studies on Gloniella (Hysteriales, Ascomycota) described by Spegazzini. Nova Hedwigia 89: 229-236.

Murillo, C., F. J. Albertazzi, J. Carranza, H. T. Lumbsch y G. Tamayo. 2009. Molecular data indicate that Rhytidhysteron rufulum (Ascomycetes, Patellariales) in Costa Rica consists of four distinct lineages corroborated by morphological and chemical characters. Mycological Research 113: 405-416.

Salinas-Salgado, E., R. Valenzuela, T. Raymundo, M. Cipriano-Salazar, B. Cruz-Lagunas y E. Hernández-Castro. 2012. Macromicetos del bosque tropical caducifolio en el municipio de Cocula, Guerrero, México. Polibotánica 34: 137-155.

Samuels, G. J. y E. Müller. 1979. Life-history studies of Brazilian Ascomycetes. 7. Rhytidhysteron rufulum and the genus Eutryblidiella. Sydowia 32: 277-292.

Sierra-López, D. 2006. Contribución al estudio de los ascomicetes bitunicados de Cataluña. Acta Botanica Barcinonensia 50: 5-434.

Ulloa, M. A. y R. T. Hanlin. 2006. Nuevo Diccionario Ilustrado de Micología. APS Press. Minnesota, USA. 672 pp. 
Vargas, F. 1979. Vegetación. Estudio del Parque Nacional Lagunas de Chacahua, Oax. Proyecto integral para el establecimiento de zona de reserva y de investigación de fauna silvestre. CARGOMO México, D.F., México. pp. 31-40.

Vargas, F. 1984. Parques Nacionales de México y Reservas Equivalentes. Pasado, presente y futuro. Colección: Grandes Problemas Nacionales. Serie: Los Bosques de México. Instituto de Investigaciones Económicas, Universidad Nacional Autónoma de México. México, D.F., México. 266 pp. 